\title{
Discharge flow of granular media from silos with a lateral orifice and injection of air
}

\author{
Pascale Aussillous ${ }^{1, \star}$, Yixian Zhou ${ }^{2,1}$, Pierre Ruyer $^{2}$, and Pierre-Yves Lagrée ${ }^{3}$ \\ ${ }^{1}$ Aix-Marseille Univ., CNRS, IUSTI UMR 7343, 13013 Marseille, France \\ ${ }^{2}$ Institut de Radioprotection et de Sûreté Nucléaire (IRSN), PSN-RES, SEMIA, LIMAR, Cadarache, St Paul-Lez-Durance, 13115, \\ France \\ ${ }^{3}$ Sorbonne Universités, UPMC Univ Paris 06, CNRS UMR 7190, Institut $\partial^{\prime}$ Alembert, F-75005 Paris, France
}

\begin{abstract}
Few studies concern the prediction of the mass flow rate of a granular media discharged from a silo with a lateral orifice. However, this situation can have pratical interest considering a tank of granular material with a leak on its side. We studied experimentally the discharge of a vertical silo filled by spherical glass beads. We consider rectangular silos with a rectangular orifice. The impact of size, aspect ratio and position of the orifice and the effect of an additional air flow were studied. The measured parameters are the mass flow rate and the pressure along the silo, whereas the controlled parameters are the size of particles, and the flow rate of air. We identified two regimes of discharge according to the aspect ratio (of width to height) of the rectangular orifice. Increasing the air flow rate induces an increase of the granular media flow rate. Using a simple physical model to describe the grains and gas flow, we put in evidence the role played by the air pressure gradient at the outlet. Then we compared the experimental results with continuum Navier-Stokes simulations with the granular $\mu(\mathrm{I})$-rheology. We showed that the continuum $\mu(\mathrm{I})$-rheology describes well our discharge flow of granular media from silos, taking into account the effect of the position of the orifice as well as the coupling with the gas flow.
\end{abstract}

\section{Introduction}

Among the wide spectrum of granular flows, we focused on a rather specific configuration: a relatively narrow elongated vertical tube, with an opening located on its lateral wall, filled with spherical glass beads, discharges its contents in a constant pressure environment, a gas flowing throughout the tube. This case is of particular interest to understand conditions under which a set of nuclear solid fuel particles inside a typical PWR fuel rod, whose cladding would have failed under hypothetical accidental conditions, could disperse out of this rod. The fuel particles are generated from an initially cylindrical pellet (scale of $1 \mathrm{~cm}$ ) that can be fragmented (the smallest size being of the order of $10 \mu \mathrm{m}$ ) due to the irradiation process (burnup of the fuel) or accidental conditions (large pressure and temperature variations inside the rod).

The flow rate of a granular medium from of a confined tank to an open space through a narrow orifice has clearly to be related to the classical hourglasses or hoppers flow rates, that have been studied since centuries. In those latter cases, the Beverloo relation [1], provides an universal scaling for the flow rate but requires some empirical constants to be applied to a specific configuration. Extension of the application range of this law to air-injection assisted [2-4], or inclined tanks have been already considered $[5,6]$. The physical interpretation of the scaling is

^e-mail: pascale.aussillous@univ-amu.fr still an active research area: as an illustrative example, the widely known concept of a free-fall arch above the orifice has been recently invalidated as a correct idealization of the near orifice granular flow [7].

This bibliographical review motivates the need for a specific investigation of the granular flow of interest. This paper presents the main results of this study. First, we present the analysis of experiments we performed thanks to a dedicated device that simulates the main aspects of such a flow configuration. Then, we considered the potential of the model of the granular medium as an equivalent continuum material with a specific rheology to correctly reproduce the experimentally observed phenomena. For this purpose, we performed numerical simulations. Using the $\mathcal{B}$ asiliskprogram, we solved the Euler-Euler two-phase mass and momentum balance equations using, (i) a simplified incompressible Darcy flow model for the gas phase and, (ii) an effective viscosity formulation to model the socalled $\mu$ (I) rheology with uniform compacity for the granular phase.

\section{Experimental study}

\subsection{Experimental device}

The silo of granular materials

Several silos have been built for this study and we will focus on the results obtained in rectangular shape tanks as 


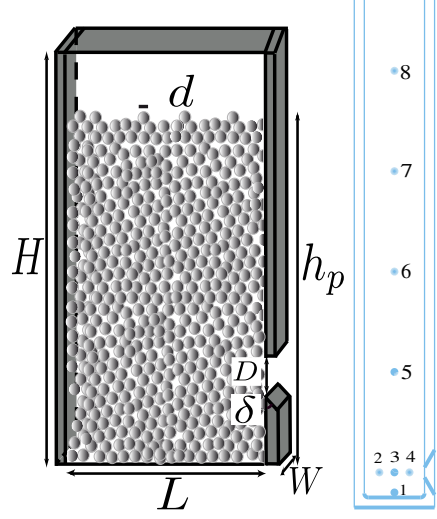

Figure 1. Schematic

illustrations of the silo. Left hand side picture: main geometrical dimensions. The wall near the orifice, of thickness $\delta$ has been bevelled till no influence of $\delta$ on the flow rate can be observed. Right hand side picture: Pressure sensors locations.

Table 1. Dimension of the silo and size of the particles

\begin{tabular}{lll}
\hline$D(\mathrm{~mm})$ & $W(\mathrm{~mm})$ & $d(\mu \mathrm{m})$ \\
\hline$[2.7,5.4,10,15, ;$ & {$[3.5,5,10$,} & {$[124,190,375,538$,} \\
$\underline{20}, 25,30,35]$ & $20,30,40]$ & $762,1129,1347]$ \\
\hline
\end{tabular}

illustrated in figure 1. The aperture on the lateral sidewall has a rectangular shape with a height $D$ and a horizontal length $W$ that corresponds to the width of the silo. The silo is initially filled with an height $h_{p}$ of monodisperse in size $d$ spherical glass beads of $\rho_{p}$ density, the initial bulk volume fraction $\phi_{b}$ being estimated. The set of dimensions of the silo and of the particles size is given in table 1, the lateral extent, resp. the height, of the silo being fixed to $L=60 \mathrm{~mm}$, resp. $H=500 \mathrm{~mm}$. More details concerning the experimental device can be found in [8].

During the discharge of the silo, the beads are collected within a recipient, its mass being measured using an electronical balance (Mettler Toldeo 6002S) with an accuracy of $0.1 \mathrm{~g}$ and a frequency of $20 \mathrm{~Hz}$. The flow rate is estimated from the time evolution of this mass and, in every case, a steady state regime is observed, the corresponding flow rate value being denoted $Q\left(\mathrm{~kg} . \mathrm{s}^{-1}\right)$ in the following.

\section{The air injection system}

For all the tests concerning the flow rate dependence to the geometry, the top of the silo is open to the atmosphere. In the tests related to the impact of an air flow on the discharge rate of granulars, this top is closed and the silo is airtight except the aperutre. An air injection system is then connected to the top of the rectangular silo. It provides a constant flow rate of air $Q_{\text {air }}\left(\mathrm{m}^{3} . \mathrm{s}^{-1}\right)$ during the discharge. Pressure has been measured at different locations within the silo along the back wall, mainly along the median vertical axis and in the near orifice region, see figure 1. Similarly to the tests with open top silo, the granular flow rate is measured and a steady state value is observed. The experiments with an air flow have been performed only for a reduced set of geometries corresponding to the underlined $D$ and $W$ values in table 1 but for the whole set of $d$ values.

\subsection{Granular flow rate as a function of geometry}

Let us consider the data collected for a given size of particles, say $d=190 \mu \mathrm{m}$ and for the whole set of $D$ and $W$

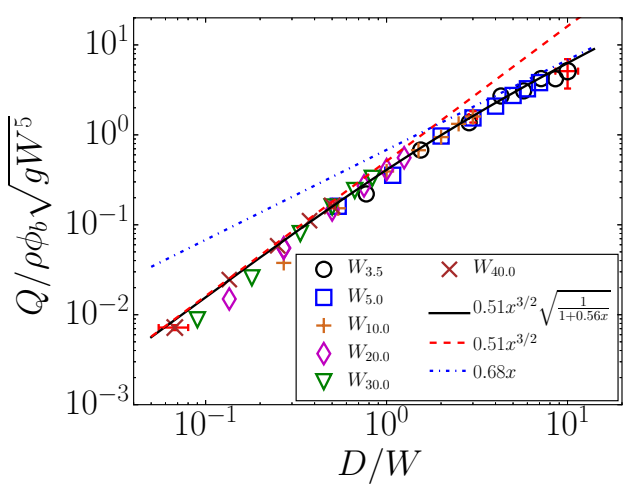

Figure 2. Experiments in the rectangular silo for a given particle diameter $d=190 \mu \mathrm{m}$ and various silo widths, $W$, the solid line represents equation $1, \sqrt{\kappa} c_{D}=0.51, \sqrt{\kappa} c_{W}=0.68$. Dashed, resp. dashed dotted lines, represents the asymptotics for $D \ll W$, resp. $W \ll D$.

values. Let us remark that, since, for this specific case, $d$ is always at least one order of magnitude smaller than the dimensions $D$ or $W$ of the aperture, one can reasonnably neglect its impact on the flow rate as suggested both by the Beverloo law or the Janda model [9]. As a preliminary study, we considered the case of discharge flow rate of similar rectangular silos, the opening being at the bottom of the silo. In the latter case, the flow rate, say $Q_{b}$, follows a single Beverloo law $Q_{b}=c_{D} D W \sqrt{g D}$. For the case of a lateral opening of interest for this study, the dimensionless flow rate $Q /\left(\rho \phi_{b} \sqrt{g W^{5}}\right)$ has been plotted for the whole set of $D$ and $W$ values on figure 2 . It is worth pointing out that the data can not be reduced to a single power law function. Actually, two clearly distinct flow regimes can be distinguished according to the $D / W$ aspect ratio of the aperture. For smallest value of this aspect ratio, i.e. when $D$ is the smallest extension of the aperture, the flow rate scales as $\rho \phi_{b} D W \sqrt{g D}$. On the other side, when $W$ is the smallest extension of the aperture, the flow rate scales as $\rho \phi_{b} D W \sqrt{g W}$. Therefore, we clearly identified two regimes according to the value of $D / W$, a behaviour that can be correctly modeled by the following relation that is illustrated on figure 2 ,

$$
\frac{Q}{\rho \phi_{b} \sqrt{\kappa g W^{5}}}=c_{D}\left(\frac{D}{W}\right)^{3 / 2} \sqrt{\frac{1}{1+\left(c_{D} / c_{W}\right)^{2} D / W}},
$$

where $0 \leq \kappa \leq 1$ corresponds to an empirical factor that reduces the driving force for the flow rate from $g$ in the classical case of a bottom orifice to $\mathrm{kg}$ in the present case of a lateral orifice. For the larger particles size, we observed that the particles size effect can be well modeled by a dilation function that depends to $d / D$ as proposed by Janda et $a l$. [9]. We recovered the same behaviour for cylindrical silos as well. Additional results can be found in [10].

\subsection{Granular flow rate as a function of air flow}

The pressure space and time evolution is as follows. The pressure remains constant while the granular materials is 


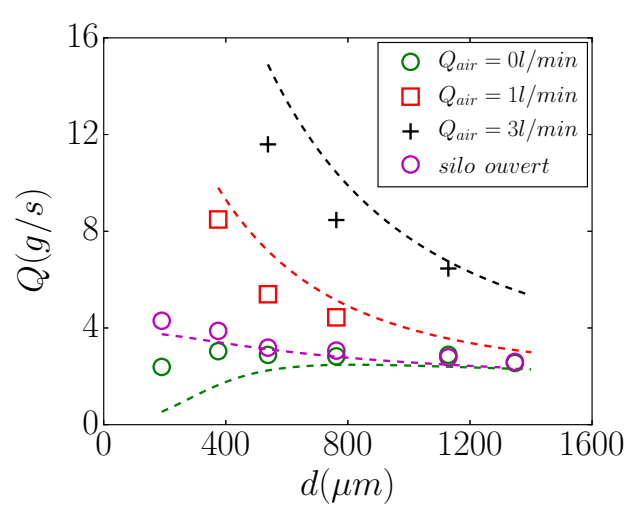

Figure 3. Rectangular silo with $D=10 \mathrm{~mm}$ : steady-state flow rate of particles as a function of particles size for different air flow rates. The solid line represents equation 3 .

above the sensor location. The pressure difference between two locations in the silo can be then successfully predicted by the Ergun model [11], for gas flow through porous media, the permeability coefficient depending on $d$ and $\phi_{b}$ and considering the density $\rho_{f}$ and the viscosity $\eta$ of the air. As soon as the sensor is located above the granular materials, the pressure gradient within the air flow is negligible and its time evolution is governed by the Ergun predicted pressure difference between the top level of the granular materials and the aperture. It is worth pointing out that the pressure gradient is larger near the orifice, that can be simply related to the acceleration of the gas due to the reduction of the flow cross section of a factor $D / L$. The permeability in such a porous medium strongly depends on the particles size. Therefore, for the same air flow rate through the beads, the pressure gradient is larger for small particles.

The granular flow rate is affected by the gas flow rate and increases with it as illustrated in figure 3. In the specific case of no air flow rate at the top of the silo, the granular flow rate is lower than for the open top case when the particles size is small. Moreover, the granular flow rate that only slightly depends on the particles size in the open top silo case, becomes all the more sensitive to the air flow rate than the particles size is small. The air flow acts clearly as an additionnal driving force for the granular flow and its impact on it follows a similar tendency than the pressure gradient throughout the granular materials.

We observed that the granular flow rate was highly correlated with the pressure gradient in a region close to the aperture of the silo that acts as a driving force in addition to gravity. Let us recall that we deduced from the open top silo experiments that the driving force for a lateral aperture was scaling the flow rate as $\sqrt{\kappa g}$. Therefore, following the models proposed in [2-4], we consider that the variation of the granular flow due to air flow can be modeled as

$$
Q=Q_{0}\left(1+\left.\frac{1}{\kappa g \phi_{b} \rho_{p}} \frac{\partial p^{f}}{\partial x}\right|_{x=x_{o}}\right)^{1 / 2}
$$

where $Q_{0}$ is the open top silo case flow rate and $\left.\frac{\partial p^{f}}{\partial x}\right|_{x=x_{o}}$ the pressure gradient near the orifice, that can be estimated thanks to the viscous $\beta_{v}=150 \phi_{b}^{2} /\left(1-\phi_{b}\right)^{3} d^{2}$ and inertial $\beta_{i}=1.75 \phi_{b} /\left(1-\phi_{b}\right)^{3} d^{2}$ terms of the permeability as predicted by Ergun's model. This leads to a rather simple analytical model to relate the non-dimensional granular flow rate $\bar{Q} \hat{=} \frac{Q}{Q_{0}}$ to the non-dimensional air flow rate $\tilde{Q}=\frac{\phi_{b} \rho_{p} Q_{a i r}}{Q_{0}}$, given the bulk volume fraction $\phi_{b}$ and the particles size $d$

$$
\begin{aligned}
\bar{Q} & =\frac{\left(-2 \mathcal{N}_{i} \tilde{Q}-\mathcal{N}_{v}\right)+\sqrt{\Delta}}{2\left(1-\mathcal{N}_{i}\right)} \\
\text { with } \Delta & =\mathcal{N}_{v}^{2}+4\left(1+\mathcal{N}_{v} \tilde{Q}\right)+4 \mathcal{N}_{v}\left(\tilde{Q}^{2}-1\right), \\
\mathcal{N}_{v} & =\frac{\eta \beta_{v}\left(\phi_{b}, d\right) Q_{0}}{\phi_{b}^{2} \rho_{p}^{2} \kappa g D W}, \text { and } \mathcal{N}_{i}=\frac{\rho_{f} d \beta_{i}\left(\phi_{b}, d\right) Q_{0}^{2}}{\phi_{b}^{3} \rho_{p}^{3} \kappa g D^{2} W^{2}}
\end{aligned}
$$

The predicted variation of this analytical model is drawn with dashed lines on the bottom graph of figure 3 . It is worth pointing out that, similarly to the cases with open top silo, we observed a deviation from the predicted results when the number of beads across the aperture $(D / d)$ is low. Once again, this behaviour can be modeled thanks to a dilation of the granular flow thanks to a geometrical function as proposed by Janda: in presence of air flow, this dilation also impacts the permeability of the granular phase in the near orifice region, an effect that can then be taken into account in our model by considering the volumetric fraction near the orifice, say $\phi_{o}$ rather than in the bulk in the hereinabove model. More detailed informations can be found in [8].

\section{Numerical simulations}

\subsection{Flow modeling and system of governing equations}

We use the classical two-phase equations (see e.g. Jackson [12] or [13]) for gaz and granular phases (with the volume average velocity ponderation of the particules and fluid velocities). This gives for each phase mass and momentum balance equations. Flows are assumed to be incompressible and steady, inertia is neglected in the momentum balance equation of the air. This leads to a Darcy flow of air through the beads. The associated pressure gradient couples both momentum balance equations. The effective viscosity formulation of the $\mu(\mathrm{I})$ rheological law with uniform volume fraction $\phi_{b}$ is used for the momentum balance equation of the grains. To solve these continuous coupled equations we use the code Basilisk an extension of the code used in [14] for dry granular flows. The rectangular silo is simulated in a $2 D$ computational domain, the silo being a $L \times H$ rectangular domain with a constant granular pressure boundary condition on the lateral orifice of size $D$. Steady air flow rate is imposed at the top of the silo.

\subsection{Taking into account the wall friction}

Let us first consider the case without air flow. To take into account the lateral friction, we average the momen- 


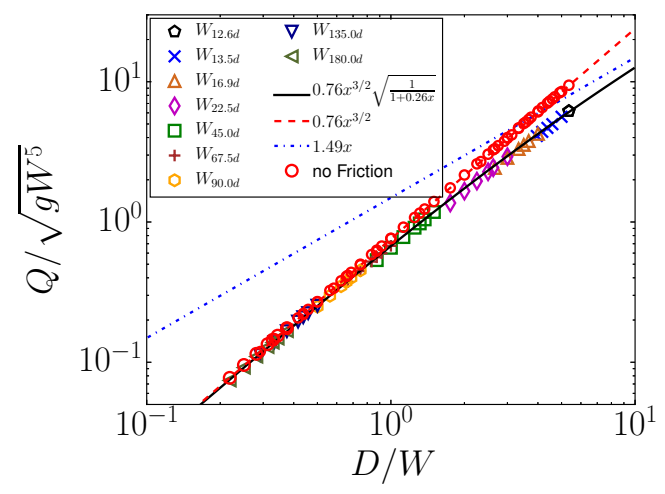

Figure 4. Numerical granular flow rate for various $D$ and $W$ values. Red circles correspond to numerical solutions of pure 2D flow, other symbols (corresponding to a given value for $W$ ) correspond to the averaged equations with the wall friction term. Plain and dashed lines correspond to the scaling laws.

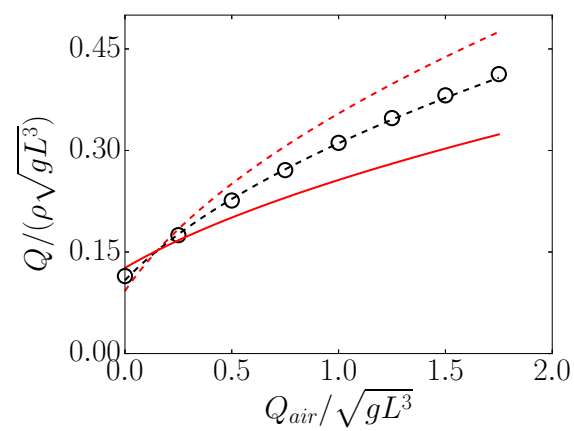

Figure 5. $Q / \rho \sqrt{g L^{3}}$ as a function of $Q_{\text {air }} / \sqrt{g L^{3}}$ for $(D=$ $0.40625 L, W=0.25 L$ ). Symbols correspond to numerical simulation. The analytical fitting use equation 3 with $(-) A=1$ and $\kappa=1,(--) A=1$ and $\kappa=0.36,(--) A=0.67$ and $\kappa=0.36$.

tum equation across the width of the silo (in the Hele-Shaw spirit, [15]). This adds an averaged additional force from the front and back sidewalls in the momentum equation. This force reads $\left(2 \mu_{w} / W\right) \mathbf{u} /\|\mathbf{u}\|$, this term being 0 for a $2 D$ case $(W=\infty)$. On figure 4 , the granular flow rate for various $D$ and $W$ values has been plotted. In the $W=\infty$ case, we recover the scaling of the flow rate in $D^{3 / 2}$, whereas for the larger $D / W$ values, the flow rate deviates from this law and tends to the experimentally observed scaling in $D W^{1 / 2}$. This result, further discussed in [10] clearly indicates that the wall friction plays a major role in the discharge flow rate from a silo having such an elongated geometry. It also extend the range of applicability of the $\mu(\mathrm{I})$ law. As a perspective, we will consider taking into account the dilation effect observed for small number of beads in the aperture $D / d$ values by solving the additional $\phi(\mathrm{I})$ rheological law.

\subsection{Air flow related additional driving force}

The symbols in the curve of figure 5 illustrates that, as observed experimentally, the numerical simulation predicts an increase discharge flow rate when air flows through the aperture. This numerical behaviour has been correctly fitted by our simple analytical model (with $\mathcal{N}_{i}=0$ for this Darcy flow) considering the reduced $k g$ driving force for the open-top case as well as a factor $A \leq 1$ in front of the fluid pressure gradient evaluated numerically at the orifice location. This latter factor is interpreted as follows: the granular flow is accelerated by the pressure gradient over a domain of $D$ extent upstream the orifice location, rather than by the pressure gradient at the orifice location, where it is maximal $(A=1)$. The ability to fit the numerical result with the model used to interpret the experimental data is an additional evidence of the actual coupling between granular and air flows during such a discharge.

\section{Conclusions}

Using experiments and continuous simulation with a granular rheology we have proposed a model which predicts the flow rate of a granular media discharging from silos with a lateral orifice in the presence of an air flow rate.

\section{References}

[1] W.A. Beverloo, H.A. Leniger, J.V. de Velde, Chem. Eng. Sci. 15, 260 (1961)

[2] J. de Jong, Q.E.J.J.M. Hoelen, Powder Technol. 12, 201 (1975)

[3] J. de Jong, Powder Technol. 3, 279 (1969)

[4] R. Nedderman, U. Tūzūn, R. Thorpe, Powder Technology 35, 69 (1983)

[5] A. Medina, D. Cabrera, A. López-Villa, M. Pliego, Powder Technology 253, 270 (2014)

[6] D. Serrano, A. Medina, G.R. Chavarria, M. Pliego, J. Klapp, Powder Technology 286, 438 (2015)

[7] S. Rubio-Largo, A. Janda, D. Maza, I. Zuriguel, R. Hidalgo, Phys. Rev. Lett. 114, 238002 (2015)

[8] Y. Zhou, Ph.D. thesis, Aix-Marseille Univ. (2016)

[9] A. Janda, I. Zuriguel, D. Maza, Phys. Rev. Lett. 108, 248001 (2012)

[10] Y. Zhou, P.Y. Lagrée, S. Popinet, P. Ruyer, P. Aussillous, submitted to J. Fluid Mech.

[11] S. Ergun, Chemical Engineering Progress 48(2), 89 (1952)

[12] R. Jackson, The Dynamics of Fluidized Particles, Cambridge Monographs on Mechanics (Cambridge University Press, 2000), ISBN 9780521781220

[13] P. Aussillous, J. Chauchat, M. Pailha, M. Médale, E. Guazzelli, J. Fluid Mech. 736, 594-615 (2013)

[14] L. Staron, P.Y. Lagrée, S. Popinet, The European Physical Journal E 37, 1 (2014)

[15] P. Jop, Y. Forterre, O. Pouliquen, J. Fluid Mech. 541, 167-192 (2005) 